\title{
Estimating the age and affinities of Homo naledi
}

AUTHOR:

J. Francis Thackeray ${ }^{1}$

\section{AFFILIATION:}

${ }^{1}$ Evolutionary Studies Institute, University of the Witwatersrand, Johannesburg, South Africa

\section{CORRESPONDENCE TO:} J. Francis Thackeray

EMAIL:

Francis.thackeray@wits.ac.za

\section{POSTAL ADDRESS:}

Evolutionary Studies Institute, Private Bag 3, Wits 2050,

South Africa

\section{KEYWORDS:}

Rising Star; Plio-Pleistocene; least squares linear regression; morphometric analysis

\section{HOW TO CITE:}

Thackeray JF. Estimating the age and affinities of Homo naledi. S Afr J Sci. 2015;111(11/12), Art. \#a0124, 2 pages. http:// dx.doi.org/10.17159/sajs.2015/ a0124 (c) 2015. The Author(s). Published under a Creative Commons Attribution Licence.
Recent discoveries of more than 1500 hominin fossils from the site of Rising Star in the South African Cradle of Humankind, attributed to a new species (Homo naledi), ${ }^{1}$ have attracted global interest. As yet no secure date for this extraordinary material has been obtained, and the relationship of this species to other Plio-Pleistocene taxa has been greatly debated in the media. Here I report results of morphometric analyses that may facilitate an assessment of the age and affinities of crania attributed to $H$. naledi.

The method is based on a least squares linear regression analysis of mean values of measurements for crania of 12 hominin species (Table 1), as published by Berger et al. ${ }^{1}$ The analyses were performed to obtain standard errors of $m$-coefficients $\left(\mathrm{se}_{\mathrm{m}}\right)$ in regression equations of the form $y=m x+c$, based on pairwise comparisons of cranial data, as described elsewhere for pairs of hominin specimens, ${ }^{2,3}$ taking into account criticisms raised by Gordon and Wood ${ }^{4}$.

The degree of scatter around a regression line of pairwise comparisons is quantified by the $\mathrm{se}_{m}$ statistic. Log transformed $\mathrm{se}_{\mathrm{m}}$ values for conspecific pairs of modern vertebrates (as well as invertebrates) display a normal distribution with a mean value of $-1.61,{ }^{5}$ which has been considered to be an approximation of a biological species constant (T) with a standard deviation of circa $0.1,{ }^{3}$ and which has been used to facilitate a mathematical (probabilistic) definition of a species ${ }^{5}$.

It is of great interest to use this approach by comparing cranial measurements of $H$. naledi to those of other species listed in Table 1, using data published by Berger et al. ${ }^{1} \mathrm{Log} \mathrm{se}_{\mathrm{m}} \mathrm{Hn}$ ( $x$ axis) values refer to results of pairwise comparisons when the measurements for $H$. naledi are on the $x$ axis, and measurements for other taxa are on the $y$ axis in regression analyses. Log $\mathrm{se}_{\mathrm{m}} \mathrm{Hn}$ ( $\mathrm{y}$ axis) values refer to results of pairwise comparisons when the measurements for $\mathrm{H}$. naledi are on the $y$ axis, and measurements for other taxa are on the $x$ axis. 'Log se mean' refers to the mean of these two

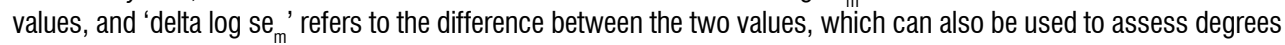
of similarity or dissimilarity in the context of $\log \mathrm{se}_{\mathrm{m}}$ values. $^{3}$

In the context of results reported for conspecific pairs of modern taxa, it is relevant to report two results from the current study. Firstly, H. naledi appears to be significantly different (dissimilar) from other species listed in Table 1 , because all of the mean log se $e_{m}$ values listed in Table 1 are outside the $95 \%$ confidence limits around the mean value of $-1.61+/-0.1$ for conspecifics. Secondly, $H$. naledi appears to be most similar to specimens attributed to early Homo, notably $H$. habilis, and (to a lesser extent) $H$. rudolfensis and $H$. erectus (see numbers listed in bold in Table 1 ).

Table 1: Results of pairwise comparisons between cranial measurements of Homo naledi and corresponding measurements of other species

\begin{tabular}{|c|c|c|c|c|}
\hline & $\begin{array}{c}\log s e_{m} \\
\text { Hn (x axis) }\end{array}$ & $\begin{array}{c}\log s e_{m} \\
\text { Hn (y axis) }\end{array}$ & $\begin{array}{c}\log s e_{m} \\
\text { mean }\end{array}$ & $\begin{array}{r}\text { Delta } \\
\log \mathrm{se}_{\mathrm{m}}\end{array}$ \\
\hline Paranthropus aethiopicus & -0.852 & -0.980 & -0.916 & 0.128 \\
\hline P. boisei & -1.098 & -1.067 & -1.082 & -0.031 \\
\hline P. robustus & -1.156 & -1.119 & -1.137 & -0.037 \\
\hline Australopithecus afarensis & -1.110 & -1.139 & -1.124 & 0.029 \\
\hline A. africanus & -1.189 & -1.142 & -1.165 & -0.047 \\
\hline A. sediba & -1.192 & -1.120 & -1.156 & -0.072 \\
\hline Homo habilis & -1.326 & -1.331 & -1.328 & 0.005 \\
\hline H. rudolfensis & -1.200 & -1.272 & -1.236 & 0.072 \\
\hline H. erectus & -1.131 & -1.256 & -1.193 & 0.125 \\
\hline H. sapiens (Middle Pleistocene) & -1.062 & -1.285 & -1.173 & 0.223 \\
\hline H. sapiens (modern) & -1.102 & -1.202 & -1.152 & 0.100 \\
\hline
\end{tabular}

Log se $e_{m} H n$ (x axis) values refer to results of pairwise comparisons when the measurements for $\mathrm{H}$. naledi are on the $\mathrm{x}$ axis, and measurements for other taxa are on the y axis. Log se ${ }_{m} H n$ (y axis) values refer to results of pairwise comparisons when the measurements for $\mathrm{H}$. naledi are on the y axis, and measurements for other taxa are on the xaxis. Log se ${ }_{m}$ mean refers to the mean of these two values, and delta $\log s e_{m}$ refers to the difference between the two values. Relatively low $\log s e_{m}$ values in bold reflect the fact that $\mathrm{H}$. naledi appears to be most similar to specimens attributed to $\mathrm{H}$. habilis, $\mathrm{H}$. rudolfensis and (to a lesser extent) $\mathrm{H}$. erectus, associated with ages in the order of $2(+/-0.5)$ mya.

A conclusion from this analysis is that the claim that $H$. naledi represents a distinct species appears to be warranted, at least from cranial data. Without assuming that $\log \mathrm{se}_{\mathrm{m}}$ values can provide accurate dates, the results presented in Table 1 may be used to provide an estimate for the age of $H$. naledi, here considered to be in the order of 2 million years $(+/-0.5$ years), recognising that the maximum age for $H$. rudolfensis is circa 2.5 mya, the age for African $H$. erectus 
in this comparative study is circa 1.5 mya, and the age for $\mathrm{H}$. habilis from sites such as Olduvai Gorge in Tanzania is circa 1.8 mya.

\section{Acknowledgements}

This research was supported by the National Research Foundation (South Africa) and the A.W. Mellon Foundation. I am grateful to Sue Dykes for assistance and discussion.

\section{References}

1. Berger LR, Hawks J, De Ruiter DJ, Churchill SE, Schmid P, Delezene LK, et al. Homo naledi, a new species of the genus Homo from the Dinaledi Chamber, South Africa. eLife. 2015;4:e09560. http://dx.doi.org/10.7554/eLife.09560
2. Thackeray JF. Homo habilis and Australopithecus africanus, in the context of a chronospecies and climatic change. In: Runge J, editor. Changing climates, ecosystems and environments within arid southern Africa and adjoining regions: Palaeoecology of Africa 33. Forthcoming 2015.

3. Thackeray JF, Dykes S. Morphometric analyses of hominoid crania, probabilities of conspecificity and an approximation of a biological species constant. HOMO J Comp Hum Biol. Forthcoming 2015.

4. Gordon AD, Wood BA. Evaluating the use of pairwise dissimilarity metrics in paleoanthropology. J Hum Evol. 2013;65:465-477. http://dx.doi. org/10.1016/j.jhevol.2013.08.002

5. Thackeray JF. Approximation of a biological species constant? S Afr J Sci. 2007;103:489 